\title{
Elevated levels of sIL-2R, TNF- $a$ and hs-CRP are independent risk factors for post percutaneous coronary intervention coronary slow flow in patients with non-ST segment elevation acute coronary syndrome
}

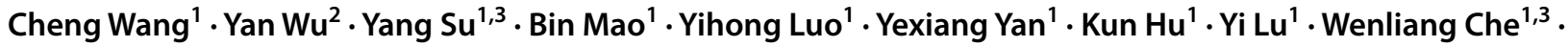 \\ Minying Wan ${ }^{1}$
}

Received: 24 August 2021 / Accepted: 12 January 2022 / Published online: 19 February 2022

(c) The Author(s) 2022

\begin{abstract}
To evaluate the association between circulating levels of inflammatory cytokines and the occurrence of post-percutaneous coronary intervention (PCI) coronary slow flow (CSF) in patients with non-ST segment elevation acute coronary syndrome (NSTE-ACS). CSF after PCI commonly occurs and implies poor outcomes, while the determinants of post-PCI CSF in patients with NSTE-ACS remain controversial. In this multicenter case control study, 176 patients diagnosed with NSTEACS and with post-PCI CSF occurred composed of CSF group, while 352 matched NSTE-ACS patients composed control group. Corrected thrombolysis in myocardial infarction frame count (cTFC), circulating levels of inflammatory cytokines and PCI related parameters were analyzed using Logistic regression models. Among 528 patients with median age of 67 (59-76) and male proportion of $65.5 \%, 176$ (35.0\%) patients had occurrence of post-PCI CSF defined as cTFC $\geq 24$. Patients with CSF presented more intense inflammatory activity revealed by higher levels of white blood cell, high-sensitivity C-reactive protein (hs-CRP), interleukin-1ß (IL-1ß), soluble IL-2 receptor (sIL-2R), IL-6, IL-8, IL-10 and tumor necrosis factor- $\alpha$ (TNF- $\alpha$ ), while PCI related parameters were comparable. Correlation analysis showed cTFC was positively correlated with those inflammatory cytokines. Logistic regression model indicates that hs-CRP (odds ratio (OR) $=3.038$, 95\% confidence interval (CI) 1.545-5.975), sIL-2R (OR $=2.103$, 95\% CI 1.959-4.026) and TNF- $\alpha(\mathrm{OR}=3.708,95 \%$ CI $1.426-9.641)$ were valuable predictors for CSF occurrence. Elevated circulating levels of inflammatory cytokine including hs-CRP, sIL-2R and TNF- $\alpha$ rather than PCI related parameters could predict post-PCI CSF in patients with NSTE-ACS.
\end{abstract}

Keywords Non-ST segment elevation acute coronary syndrome (NSTE-ACS) · Coronary slow flow (CSF) · Percutaneous coronary intervention $(\mathrm{PCI}) \cdot$ Inflammatory cytokines

Cheng Wang and Yan Wu have contributed equally to this work and share first authorship.

Wenliang Che

chewenliang@tongji.edu.cn

$\triangle$ Minying Wan

2679384102@qq.com

1 Department of Cardiology, Chongming Branch, Shanghai Tenth People's Hospital, Tongji University School of Medicine, 66 East Xiangyang Road, Chongming, Shanghai, China

2 Department of Cardiology, Shanghai Putuo District Liqun Hospital, Shanghai 200333, China

3 Department of Cardiology, Shanghai Tenth People's Hospital, Shanghai 200072, China

\section{Introduction}

Coronary slow flow (CSF) is a common phenomenon in acute coronary syndrome (ACS) patients, presenting as significant deceleration of coronary blood flow. Compared with non-CSF, patients with CSF have a higher incidence of major adverse cardiovascular events (MACE) during the follow-up period. Furthermore, post-percutaneous coronary intervention (post-PCI) CSF should draw additional attention due to the occurrence of microvascular thrombosis and/or endothelial dysfunction that contributes to worse outcomes [1]. According to literatures, the incidence of post-PCI CSF is $14-25 \%$ in patients with ST-segment elevation myocardial infarction (STEMI) [2]. Previous studies among patients 
with STEMI have showed that hyperglycemia, longer reperfusion time, higher stent to vessel diameter ratio and heavier thrombus burden independently predict post-PCI CSF [3]. However, plaque morphology of non-ST segment elevation acute coronary syndrome (NSTE-ACS) differs from that of STEMI, which is characterized by thicker fibrous cap, more calcified nodules, more intensively diffused atherosclerosis and milder thrombus burden [4]. Therefore, the risk factors for post-PCI CSF in patients with NSTE-ACS could be disparate from those for STEMI and should be independently evaluated. Previous studies have showed that determinants of post-PCI CSF in patients with NSTE-ACS are multifarious, including metabolic indices as BMI, lifestyle factors as smoking status [5], serological biomarkers such as biomarkers of cardiac injury [6], fibrinogen, ischemic modified albumin [7], homocysteine [8], soluble adhesion molecules [9] and choline [10]. Throughout published literatures, determinants of post-PCI CSF in patients with NSTE-ACS has remained controversial.

Inflammatory response plays a crucial role in the development of atherosclerosis, especially in culprit or non-culprit plaques in patients with ACS [11]. Mounting evidences have supported that inflammation is a central pathogenetic process of the progression of atherosclerosis, formation of unstable plaque as well as plaque rupture [12]. The more intense inflammatory response is, the more easily atheroma eruption and microvascular spasm occur. While circulating level of cytokines could indicate the intensity of inflammation, especially TNF- $\alpha$ and interleukins that participate in endothelial dysfunction, inflammatory invasion and development of unstable atherosclerotic plaque [13-17]. Therefore, we suppose that circulating inflammatory cytokines could act as important predictors for CSF phenomenon. The present retrospective cohort study is designed to reveal the expression patterns of inflammatory cytokines in patients with NSTE-ACS, and validate the predictive value of these cytokines for post-PCI CSF phenomenon.

\section{Materials and methods}

\section{Study population}

In this multi-center case-control study, data of patients who diagnosed with NSTE-ACS and underwent PCI procedure were collected from 3 centers including Shanghai Tenth People's Hospital, Chongming Branch of Shanghai Tenth People's Hospital and Putuo District Liqun Hospital from February 2014 to January 2016. The study was approved by the Ethics Committee of Shanghai Tenth People's Hospital (SHSY-IEC-4.1/20-139/01), the Ethics Committee of Chongming Branch of Shanghai Tenth People's Hospital (SYCM-YJKT-20-0814/01) and the Ethics Committee of Putuo District Liqun Hospital (RT-202013). Written informed consent for blood sample detection and medical treatment was acquired from each patient on admission.

The inclusion criteria were (1) $\geq 18$ years old, (2) patients diagnosed as NSTE-ACS according to 2015 ESC guideline [18], and (3) patients required to undergo revascularization according to 2014 ESC/EACTS guideline on myocardial revascularization [19]. The exclusion criteria were (1) severe liver or kidney diseases, (2) trauma, infection and surgery within past three months.

CSF group (case group) included 176 NSTE-ACS patients with the occurrence of post-PCI CSF, excluding 46 patients received peripheral and/or intracoronary injection of IIb/ IIIa inhibitor (tirofiban), 26 patients with insufficient quality of angiographic images and 18 patients with unqualified blood sample. While 352 patients diagnosed with NSTEACS and underwent revascularization but without post-PCI CSF occurred were included as control group that was 1:2 matched. A loading dose of antiplatelet drugs (either aspirin $300 \mathrm{mg}$ + clopidogrel $300 \mathrm{mg}$ or aspirin $300 \mathrm{mg}+$ ticagrelor $180 \mathrm{mg}$ ) was given to the patients before PCI if the patient hasn't taken any antiplatelet drug before.

\section{Data collection}

Blood samples were obtained for routine lab tests and specific inflammatory cytokines detection within $24 \mathrm{~h}$ since admission and before any treatment including medication and primary PCI. Soluble interleukin-2 receptor (sIL-2R), interleukin-8 (IL-8), interleukin-10 (IL-10) and tumor necrosis factor- $\alpha$ (TNF- $\alpha$ ) were measured using ELISA kits (Xinyu Biological Technology Co., Ltd. Shanghai, China). High-sensitivity C-reactive protein (hs-CRP) was measured by a Roche Tina-quant immuno-turbidimetric assay (Roche Diagnostics). Interleukin-1 $\beta$ (IL-1 $\beta$ ) and interleukin-6 (IL6) was measured using an EV3513 cytokine biochip array (Randox Laboratories, Crumlin, UK) and competitive chemiluminescence immunoassays (Randox Laboratories, Crumlin, UK).

Demographic information, physical examination, medical history, electrocardiogram and echocardiography were acquired from electronic records. Film and video data of angiography and PCI were collected for analysis and reviewed by two proficient investigators individually. Measurement of imageological data right after revascularization and before any medication (nitrates, adenosine, calcium antagonist or IIb/IIIa antagonist) was accomplished in order to evaluate the occurrence of CSF. 


\section{Images measurements}

All coronary angiography evaluation after PCI was performed using $5 \mathrm{~F}$ catheter, and images were obtained at the rate of 30 frames per second. Lesion type was classified according to previous study [20]. Haziness was defined as the presence of inhomogeneous contrast and/or indistinct vessel borders and filling defect was defined as the presence of intraluminal region with no filling of contrast [21]. Representative images were displayed in Supplementary Fig. 1. In the situation of multivessel disease, the coronary artery with the most severe lesion was selected for CSF evaluation. When more than one stent was needed for the candidate vessel, the stent diameter was defined as the mean diameter of stents measured after post-dilation and the stent length was the sum of length of implanted stents. $\mathrm{Sd} / \mathrm{RVd}$ ratio was calculated by the ratio of stent diameter and reference vessel diameter, which assessed the effectiveness of stent implantation [22]. Coronary stenosis was measured using quantitative coronary angiography (QCA) method performed on digital subtraction angiography (DSA) workstation (Siemens, German). Quantitative flow ratio (QFR) after revascularization was measured using AngioPlus (Pulse Medical Imaging Technology, Shanghai, China) based on two different angiographic image views with angel $\geq 25^{\circ}$. CSF was defined as corrected thrombolysis in myocardial infarction (TIMI) frame count (cTFC) $\geq 24$ [23]. The cTFC of left anterior descending (LAD) artery was divided by 1.7 to generate an adjusted value due to the longer anatomic length than the other two major arteries. Total Syntax score for each participant was calculated using the SYNTAX score calculator (version 2.28, www.syntaxscore.com).

\section{Statistical analysis}

Statistical analysis was conducted using SPSS 22.0 (IBM Inc., USA). Continuous variables were presented as mean \pm standard deviation (SD) or median with interquartile range (IQR), and compared using 2-sample t-test if they conform normal distribution or Mann-Whitney $U$-test if not. Dichotomous variables were presented as proportion and compared using Fisher exact test or $\chi^{2}$ test.

Spearman correlation analysis was conducted to explore the association between the CTFC and measured variables. Receiver operator characteristics (ROC) analysis was conducted to evaluate the generate cut-off value of inflammatory cytokines. Furthermore, univariate and multivariate Logistic regression models were built to explore risk factors of CSF. Inflammatory cytokines were incorporated in continuous or binary forms in the multivariate model respectively. The multivariate Logistic model was adjusted for gender, age, fasting blood glucose (FBG), diastolic blood pressure (DBP), heart rate (HR), cardiac troponin $\mathrm{T}$ (cTnT), total cholesterol (TC), high density lipoprotein (HDL), stent length and post dilation pressure. All tests were two-sided and $\mathrm{p}<0.05$ was considered statistically significant.

\section{Patient and public involvement}

The patients, the public or any third parties were not involved in the design, conduct, reporting or dissemination of our research.

\section{Results}

\section{Baseline clinical characteristics of patients with CSF}

Overall, 528 patients with $65.5 \%$ male and median age of 67 [59-76] were eligible to be finally included. The inflammatory cytokines concentration distribution in overall population was displayed in Supplementary Fig. 2. The demographic feature and medical history were comparable between CSF and control groups (Table 1). In CSF group, patients displayed lower level of hemoglobin $(\mathrm{Hb})$, and higher level of white blood cell count (WBC), N-terminal pro B-type natriuretic peptide (NT-proBNP), total cholesterol (TC), fast blood glucose (FBG), high sensitivity C-reactive protein (hs-CRP), interleukin-1 $\beta$ (IL-1 $\beta$ ), soluble interleukin-2 receptor (sIL-2R), interleukin-6 (IL-6), interleukin-8 (IL-8), interleukin-10 (IL-10) and tumor necrosis factor- $\alpha(\mathrm{TNF}-\alpha)$. Likewise, there are no difference in medical history and antiaggregant loading drugs using between groups (Supplementary Table 1). This probably indicate that patients with CSF occurrence after PCI usually had metabolic disturbance and more active inflammatory responses.

\section{Higher inflammatory level appeared in CSF patients subdivided by culprit vessels}

The expression level of each cytokine was further explored according to culprit vessels (Fig. 1). Generally, overall cytokines level had no significant difference among 3 culprit vessels except the level of TNF- $\alpha$ was lower in subgroup of LAD compared with that of RCA. While patients with CSF had a higher level of inflammatory cytokine than non-CSF patients within each culprit vessel subgroup, except IL-1 $\beta$ level and sIL-2R in LCX subgroup, and IL-6 in RCA subgroup showed no significant difference between CSF and non-CSF patients. Totally, most of the inflammatory cytokines present 
Table 1 Baseline characteristics and inflammatory cytokine levels in subgroups

\begin{tabular}{|c|c|c|c|c|}
\hline & $\begin{array}{l}\text { Overall } \\
(\mathrm{N}=528)\end{array}$ & $\begin{array}{l}\text { Non-CSF } \\
(\mathrm{N}=352)\end{array}$ & $\begin{array}{l}\text { CSF } \\
(N=176)\end{array}$ & P-value \\
\hline Age (years) & $67(59-76)$ & $67(60-76)$ & $67(59-76)$ & 0.812 \\
\hline Male/female (n/n) & $346 / 182$ & $214 / 138$ & $132 / 44$ & 0.093 \\
\hline HR (bpm) & $70(61-75)$ & $68(61-75)$ & $71(65-76)$ & 0.109 \\
\hline $\mathrm{SBP}(\mathrm{mmHg})$ & $130(120-143)$ & $128(119-141)$ & $132(121-143)$ & 0.146 \\
\hline DBP (mmHg) & $73(62-87)$ & $76(63-88)$ & $69(61-85)$ & 0.116 \\
\hline DM, n (\%) & $156(29.5)$ & $100(28.3)$ & $56(31.8)$ & 0.768 \\
\hline HTN, n (\%) & $334(63.3)$ & $212(60.2)$ & $122(69.3)$ & 0.322 \\
\hline TC (mmol/l) & $3.19(1.55-4.36)$ & $2.85(1.24-3.97)$ & $3.43(1.90-4.59)$ & $0.013^{*}$ \\
\hline $\mathrm{TG}(\mathrm{mmol} / \mathrm{l})$ & $1.73(1.11-2.43)$ & $1.48(1.08-2.47)$ & $1.85(1.36-2.42)$ & 0.209 \\
\hline HDL (mmol/l) & $1.21(1.02-1.4)$ & $1.27(1.02-1.45)$ & $1.185(1.04-1.35)$ & 0.140 \\
\hline $\mathrm{LDL}(\mathrm{mmol} / \mathrm{l})$ & $2.33 \pm 0.77$ & $2.38 \pm 0.78$ & $2.24 \pm 0.75$ & 0.205 \\
\hline LP(a) (mg/dl) & $18.9(11.4-35.8)$ & $19.9(11.9-35.1)$ & $17.2(9.9-33.4)$ & 0.143 \\
\hline WBC (/nl) & $6.8(5.7-8.2)$ & $6.4(5.6-7.7)$ & $7.4(5.9-8.8)$ & $0.023^{*}$ \\
\hline $\mathrm{Hb}(\mathrm{g} / \mathrm{l})$ & $131.6 \pm 16.8$ & $133.4 \pm 14.4$ & $128.4 \pm 20.1$ & $0.036^{*}$ \\
\hline $\mathrm{FBG}(\mathrm{mmol} / \mathrm{l})$ & $4.9(4.2-5.7)$ & $4.6(3.7-5.6)$ & $5.0(4.5-5.8)$ & $0.019 *$ \\
\hline $\mathrm{HbA1c}(\%)$ & $6(5.6-6.6)$ & $5.9(5.6-6.4)$ & $6.2(5.7-7.4)$ & 0.050 \\
\hline cTnT (ng/ml) & $17.9(2.1-36.3)$ & $19.0(2.9-33.8)$ & $9.0(1.7-41.6)$ & 0.470 \\
\hline CK-MB (ng/ml) & $10.1(1.8-28.4)$ & $2.9(1.5-25.2)$ & $21.9(4.1-37.3)$ & $<0.001$ \\
\hline NT-proBNP (mmol/l) & $135.8(60.4-552.6)$ & $122.5(60.4-400.6)$ & 202.6 (61.9-974.0) & $0.028 *$ \\
\hline $\mathrm{eGFR}\left(\mathrm{ml} /\left(\min * 1.73 \mathrm{~m}^{2}\right)\right)$ & $96.0 \pm 32.4$ & $99.0 \pm 31.9$ & $91.1 \pm 33.4$ & 0.095 \\
\hline BUN (mmol/l) & $5.8(4.8-7)$ & $5.9(4.8-6.8)$ & $5.7(4.9-7.5)$ & 0.302 \\
\hline $\mathrm{UA}(\mu \mathrm{mol} / \mathrm{l})$ & $326.9 \pm 100.8$ & $324.5 \pm 95.7$ & $331.0 \pm 109.7$ & 0.656 \\
\hline D-dimer (mg/l) & $0.11(0.07-0.17)$ & $0.11(0.07-0.17)$ & $0.11(0.08-0.19)$ & 0.438 \\
\hline hs-CRP (mg/l) & $2.3(1.1-5.9)$ & $3.02(2.3-3.2)$ & $3.1(3.0-8.4)$ & $<0.001^{*}$ \\
\hline IL-1 $\beta(\mathrm{pg} / \mathrm{ml})$ & $0.63(0.40-1.51)$ & $0.53(0.40-1.28)$ & $1.21(0.60-2.12)$ & $0.001 *$ \\
\hline sIL-2R (U/ml) & $437.0(351.0-534.5)$ & $416.0(343.5-510.0)$ & $468.5(371.8-562.0)$ & $0.012 *$ \\
\hline IL-6 (pg/ml) & $4.01(2.56-7.71)$ & $3.44(2.47-6.17)$ & $5.77(3.32-9.71)$ & $0.002 *$ \\
\hline IL-8 (pg/ml) & $27.7(14.3-75.9)$ & $22.5(12.1-55.3)$ & $52.7(18.8-105.0)$ & $<0.001 *$ \\
\hline IL-10 (pg/ml) & $1.3(0.9-2.0)$ & $1.2(0.8-1.5)$ & $1.7(1.2-4.0)$ & $0.003^{*}$ \\
\hline TNF- $\alpha(\mathrm{pg} / \mathrm{ml})$ & $30.3(16.6-67.5)$ & $24.1(14.3-52.5)$ & $50.3(28.1-88.7)$ & $<0.001 *$ \\
\hline
\end{tabular}

Continuous variables are presented as the mean \pm standard deviation if they conform to a normal distribution, and otherwise as the median with interquartile range. Categorical variables are presented as $\mathrm{n}(\%)$

$C S F$ coronary slow flow, $H R$ heart rate, $S B P$ systolic blood pressure, $D B P$ diastolic blood pressure, $D M$ diabetes mellitus, HTN hypertension, $T C$ total cholesterol, $T G$ total triglyceride, $H D L-C$ high-density lipoprotein cholesterol, $L D L-C$ low-density lipoprotein cholesterol, $L P(a)$ lipoprotein (a), $W B C$ white blood cells, $H b$ hemoglobin, $F B G$ fast blood glucose, $H b A l C$ glycated hemoglobin A1C, $c T N T$ cardiac troponin T, $C K-M B$ creatinine kinase-myocardial band, NT-proBNP N-terminal pro B-type natriuretic peptide, $e G F R$ estimated glomerular filtration rate, $B U N$ blood urea nitrogen, $U A$ uric acid, $h s-C R P$ high sensitivity C-reactive protein, $I L-1 \beta$ interleukin- $1 \beta, s I L-2 R$ soluble interleukin-2 receptor, $I L-6$ interleukin-6, $I L-8$ interleukin-8, $I L-10$ interleukin-10, TNF- $\alpha$ tumor necrosis factor- $\alpha$

$* \mathrm{P}$ value $<0.05$. There is significant difference between groups 
Fig. 1 Difference in expression level of inflammatory cytokines and incidence of CSF among patients grouped by culprit artery. $*$ Indicates $\mathrm{P}$ value $<0.05, * *$ indicates $\mathrm{P}$ value $<0.01$. We performed comparisons of a inflammatory cytokine levels between CSF patients and non-CSF patients in each culprit vessel, $\mathbf{b}$ inflammatory cytokine levels of CSF patients among different culprit vessels, and $\mathbf{c}$ overall levels of inflammatory cytokine among patients with different culprit vessels (a)

hs-CRP

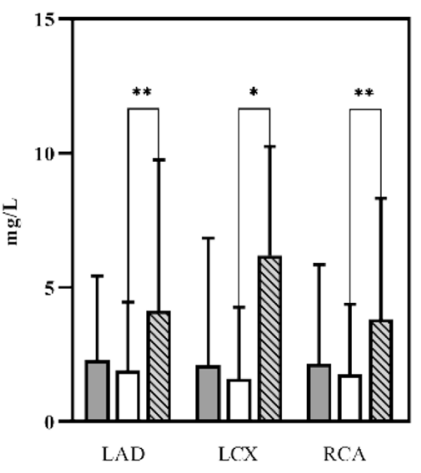

(c)

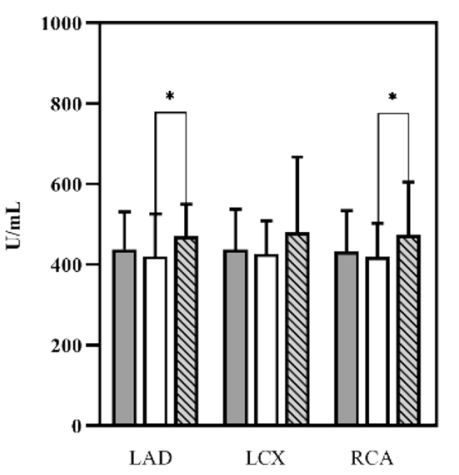

(e)

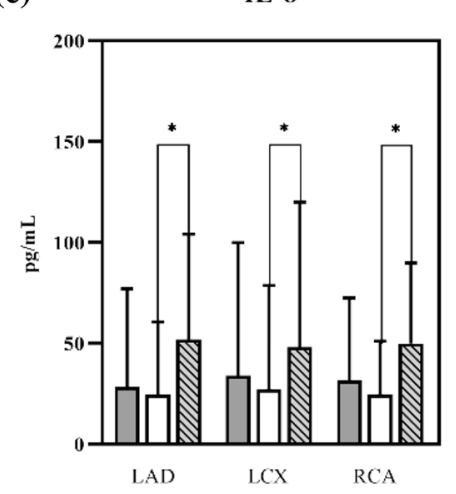

(g)

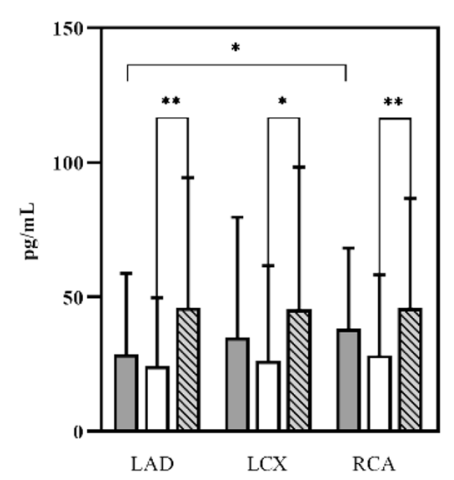

(b)

IL-1;

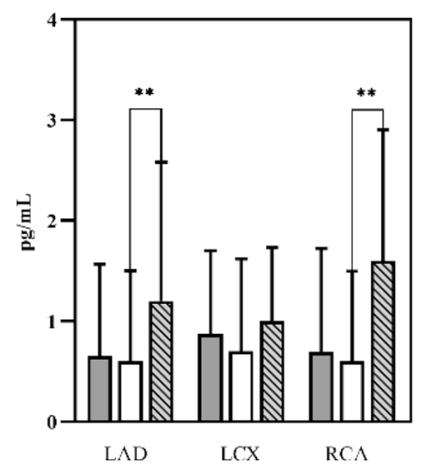

(d)

IL-6

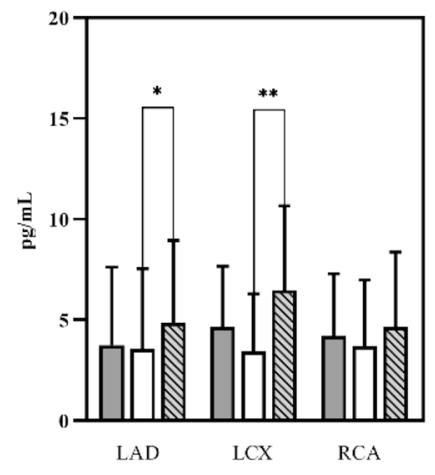

(f)

IL-10

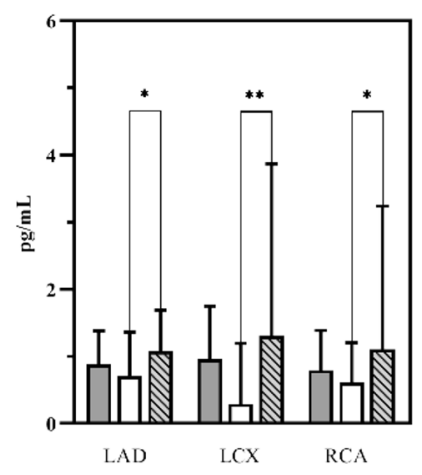

(h)

Incidence of CSF

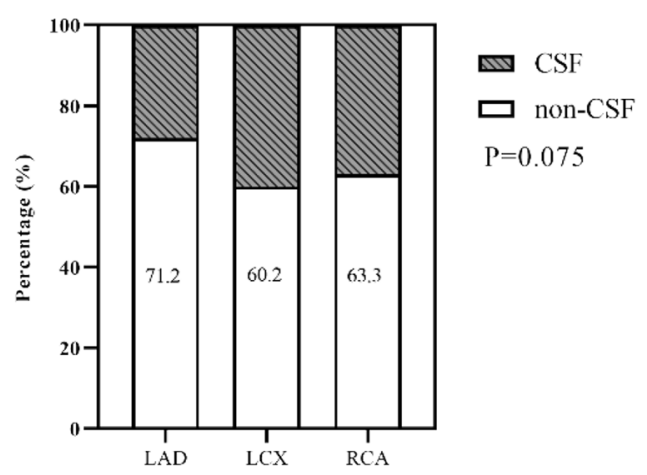


Table 2 Comparison of angiographic findings and PCI related parameters

\begin{tabular}{|c|c|c|c|c|}
\hline & $\begin{array}{l}\text { Overall } \\
(\mathrm{N}=528)\end{array}$ & $\begin{array}{l}\text { Non-CSF } \\
(\mathrm{N}=352)\end{array}$ & $\begin{array}{l}\text { CSF } \\
(N=176)\end{array}$ & P-value \\
\hline \multicolumn{5}{|c|}{ Angiographic findings before revascularization } \\
\hline Culprit vessel & & & & 0.075 \\
\hline $\mathrm{LAD}, \mathrm{n}(\%)$ & $267(50.6)$ & $190(54.0)$ & $77(43.8)$ & \\
\hline LCX, n (\%) & $103(19.5)$ & $62(17.6)$ & $41(23.3)$ & \\
\hline RCA, n (\%) & $158(29.9)$ & $100(28.4)$ & $58(33.0)$ & \\
\hline Number of diseased vessels & & & & 0.075 \\
\hline 1 vessel, n (\%) & $184(34.8)$ & $134(38.1)$ & $50(28.4)$ & \\
\hline 2 vessels, $\mathrm{n}(\%)$ & $145(27.5)$ & $90(25.6)$ & $55(31.3)$ & \\
\hline 3 vessels, $\mathrm{n}(\%)$ & $191(36.2)$ & $122(34.7)$ & $69(39.2)$ & \\
\hline Syntax score & $19.5(12.0-26.0)$ & $20.0(11.5-24.3)$ & $18.0(13.8-29.1)$ & 0.386 \\
\hline \multicolumn{5}{|l|}{ Culprit lesion feature } \\
\hline Lesion type & & & & 0.405 \\
\hline $\mathrm{A}$ and $\mathrm{B} 1, \mathrm{n}(\%)$ & $86(16.3)$ & $54(15.3)$ & $32(18.2)$ & \\
\hline $\mathrm{B} 2$ and $\mathrm{C}, \mathrm{n}(\%)$ & $442(83.7)$ & $298(84.7)$ & $144(81.8)$ & \\
\hline Bifurcation, n (\%) & $52(9.8)$ & $39(11.1)$ & $13(7.4)$ & 0.179 \\
\hline Calcified lesion, n (\%) & $124(23.5)$ & $76(21.6)$ & $48(27.3)$ & 0.147 \\
\hline Haziness, n (\%) & $47(8.9)$ & $23(6.5)$ & $24(13.6)$ & 0.001* \\
\hline Filling defect, n (\%) & $9(1.7)$ & $2(0.6)$ & $7(4.0)$ & 0.013* \\
\hline cTFC & $21(17-26)$ & $19(16-21)$ & $27(25-30)$ & $<0.001 *$ \\
\hline \multicolumn{5}{|l|}{ PCI related parameters } \\
\hline $\mathrm{Sd} / \mathrm{RVd}$ ratio & $0.86 \pm 0.08$ & $0.85 \pm 0.08$ & $0.87 \pm 0.08$ & 0.169 \\
\hline Stent diameter $(\mathrm{mm})$ & $3(2.75-3.5)$ & $3(2.75-3.5)$ & $3.25(2.94-3.5)$ & 0.150 \\
\hline Stent length (mm) & $24(18-33)$ & $24(18-33)$ & $23(17-30)$ & 0.428 \\
\hline Single stent, n (\%) & $179(82.5)$ & $115(85.2)$ & $64(84.2)$ & 0.850 \\
\hline Post-dilation pressure (atm) & $14(11-18)$ & $14(10-17)$ & $15(12-19)$ & 0.255 \\
\hline Post-dilation counts (n) & $2(1-4)$ & $2(1-4)$ & $3(1-4)$ & 0.219 \\
\hline Post-PCI QFR & $1(0.87-1)$ & $1(0.88-1)$ & $1(0.86-1)$ & 0.304 \\
\hline
\end{tabular}

Continuous variables are presented as the mean \pm standard deviation if they conform to a normal distribution, and otherwise as the median with interquartile range. Categorical variables are presented as $\mathrm{n}(\%)$

$C S F$ coronary slow flow, $L A D$ left anterior descending artery, $L C X$ left circumflex artery, $R C A$ right coronary artery, $S d / R V d$ ratio stent diameter/reference vessel diameter, $P C I$ percutaneous intervention, $Q F R$ quantitative flow ratio, $c T F C$ corrected thrombolysis in myocardial infarction frame count

$* \mathrm{P}$ value $<0.05$. There is significant difference between groups higher level in CSF patients regardless of culprit vessels, and the incidence of CSF was comparable among coronaries.

\section{Angiographic findings and $\mathrm{PCl}$ related parameters}

The peri-procedure data were analyzed between groups as displayed in Table 2. The median cTFC of patients with CSF and non-CSF was 27 [25-30] and 19 [16-21] respectively. CSF occurrence presented comparably among 3 culprit vessels. The severity and complexity of the coronary lesion between groups were similar, measured by number of diseased vessels, syntax score, lesion type and calcified lesion. While the appearance of angiographic haziness and filling defect in CSF patients was significantly higher $(13.6 \%$ vs. $6.5 \%$, P-value $=0.001 ; 4.0 \%$ vs.
$0.6 \%$, P-value $=0.013$, respectively). Focusing on the relevant parameters in PCI procedure, there were no significant difference between groups on stent length, the proportion of singlestent treatment, post-dilation pressure and post-dilation counts. Besides, the geometric and functional results after revascularization was comparable, indicated by $\mathrm{Sd} / \mathrm{RVd}$ ratio and postPCI QFR. As Table 2 presented, there were no significant differences in lesion feature and procedure details between CSF and control groups except haziness and filling defect. 
Table 3 cTFC positively correlated with inflammatory cytokines

\begin{tabular}{lclllc}
\hline & r & P value & & r & P value \\
\hline Hb & 0.157 & 0.220 & WBC & 0.210 & 0.081 \\
FBG & 0.086 & 0.214 & hs-CRP & 0.272 & $<0.001^{*}$ \\
TC & 0.160 & $0.042^{*}$ & IL-1 $\beta$ & 0.200 & $0.026^{*}$ \\
NT-proBNP & 0.127 & 0.066 & sIL-2R & 0.199 & $0.004^{*}$ \\
Sd/RVd ratio & 0.063 & 0.361 & IL-6 & 0.215 & $0.004^{*}$ \\
Post-PCI QFR & -0.068 & 0.324 & IL-8 & 0.148 & $0.032^{*}$ \\
Syntax score & 0.044 & 0.524 & IL-10 & 0.207 & $0.021^{*}$ \\
Stent length & 0.019 & 0.781 & TNF- $\alpha$ & 0.240 & $<0.001^{*}$ \\
\hline
\end{tabular}

Spearman correlation analysis were performed to test the correlation between corrected thrombolysis in myocardial infarction frame count (cTFC) and blood glucose, blood lipid, lesion severity, stenting procedure and inflammatory factors. cTFC was positively correlated with hs-CRP, IL-1 $\beta$, sIL-2R, IL-6, IL-8, IL-10, TNF- $\alpha$ and TC

$H b$ hemoglobin, $F B G$ fast blood glucose, $T C$ total cholesterol, $N T$-proBNP N-terminal pro B-type natriuretic peptide, $S d / R V d$ ratio stent diameter/reference vessel diameter, $P C I$ percutaneous intervention, $Q F R$ quantitative flow ratio, $h s$ - $C R P$ high sensitivity C-reactive protein, $I L-1 \beta$ interleukin- $1 \beta, s I L-2 R$ soluble interleukin- 2 receptor, $I L-6$ interleukin-6, $I L-8$ interleukin-8, $I L-10$ interleukin-10, $T N F-\alpha$ tumor necrosis factor- $\alpha$

$* \mathrm{P}$ value $<0.05$, the correlation is significant

\section{Correlations between cTFC and inflammatory cytokines}

To explore the relevant factors of coronary blood flow velocity presented by cTFC, Spearman correlation analysis were conducted. As Table 3 showed, 8 factors revealed positive correlation with cTFC, including TC $(\mathrm{r}=0.160)$, hs-CRP $(r=0.272)$, IL-1 $\beta(r=0.200)$, sIL-2R $(r=0.199)$, IL-6 $(r=0.215)$, IL-8 $(r=0.148)$, IL-10 $(r=0.207)$ and TNF- $\alpha$ $(r=0.240)$. However, there was no correlation between cTFC and blood cell counts, glucose metabolism, cardiac condition and PCI related parameters. Hence, the circulating level of these inflammatory cytokines might positively stand for the severity of post-PCI CSF phenomenon.

\section{Inflammatory cytokines act as risk factors of post-PCI CSF}

The cutoff values of inflammatory cytokines were calculated from ROC analysis for further variables dichotomy (Fig. 2). To explore potential risk factors of post-PCI CSF, univariate Logistic regression model showed that cTNT and inflammatory cytokines including hs-CRP, sIL-2R and TNF- $\alpha$ were predictor variables of post-PCI CSF. After adjustment for confounding factors, hs-CRP and TNF- $\alpha$ showed predictive value for post-PCI CSF (Fig. 3a). Furthermore, variables of inflammatory cytokines were divided into dichotomous variables by cutoff values. The new multivariate Logistic regression model indicated that higher level than cut-off values of hs-CRP $(\mathrm{OR}=3.038, \mathrm{P}$-value $=0.001)$, sIL-2R $(\mathrm{OR}=2.103$, P-value $=0.025)$ and $\mathrm{TNF}-\alpha(\mathrm{OR}=3.708$, $\mathrm{P}$-value $=0.007)$ could not only independently predict the occurrence of post-PCI CSF but also presented stronger predictive value (Fig. 3b).

\section{Discussion}

This is the first study comprehensively evaluated the expression pattern of hs-CRP, IL-1ß, sIL-2R, IL-6, IL-8, IL-10 and TNF- $\alpha$, and explored the predictive value of baseline level of those inflammatory cytokine for post-PCI CSF phenomenon in a NSTE-ACS cohort. Compared with nonCSF, the level of inflammatory cytokines was significantly elevated in patients suffered from CSF after revascularization, indicating the more intense inflammatory response in the population. Besides, the positive correlation between level of inflammatory cytokines and cTFC indicates that those cytokines could reflect the severity of CSF. Notably, it is the level of 3 inflammatory cytokines, hs-CRP, sIL-2R and TNF- $\alpha$, rather than other factors including metabolic disturbance, severity of myocardium injury, complexity of coronary lesion or PCI related parameters that showed independent predictive value for the occurrence of post-PCI CSF.

Of note, the comparison of angiographic findings shows that patients with CSF have a higher incidence of haziness and filling defect. Previous studies using intracoronary imaging modalities show that haziness and filling defect on angiography could be resulted from miscellaneous causes including plaque rupture, thrombosis and dissection revealed by IVUS or Swiss cheese appearance by OCT [24]. Therefore, angiographic findings alone provide only limited information, which could not comprehensively reveal the feature of underlying lesion. Owning to the limited sample size of our study, the association of haziness/filling defect and CSF needs to be investigated in future studies.

Despite CSF occurred less frequently in patients with NSTE-ACS compared STEMI, this phenomenon should still draw our attention owning to its significant contribution to adverse outcomes [25, 26]. Undoubtedly, it is of great importance to ascertain the predictors for the occurrence of post-PCI CSF. In patients with primary CSF, defined as slow coronary filling in absence of stenosis, CTFC has been confirmed to be positively correlated with multitudes of inflammatory cytokines including hs-CRP, IL-6 [27, 28] and Interferon- $\gamma$ receptor 1 [29]. In patients with NSTEMI, elevated lipoprotein-associated phospholipase A2 which acts as a vascular specific inflammatory cytokine could predict post-PCI CSF occurrence [30]. Besides, a small amount of study reported that adipocytokines played a protective role in patients with CAD who experienced CSF [31, 32]. In accord 
Fig. 2 Cut-off values of inflammatory cytokines analyzed by ROC curves. ROC curves were conducted to calculate the cutoff values of each cytokine

\section{IL-1 $\beta$}

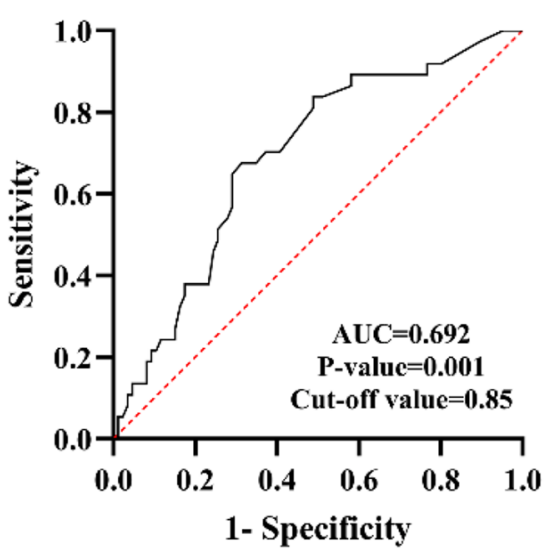

IL-6

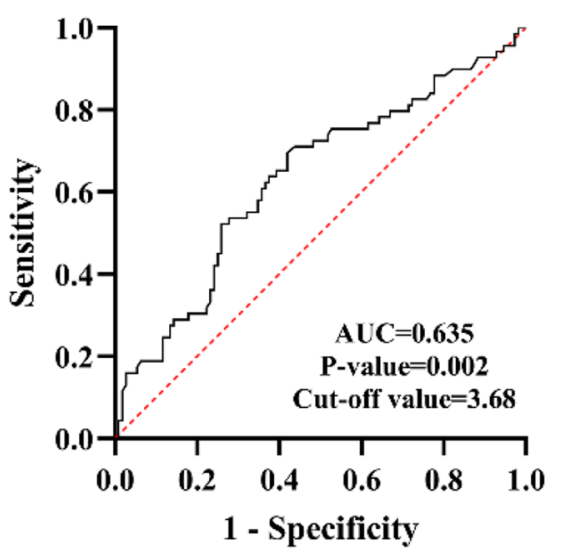

IL-10

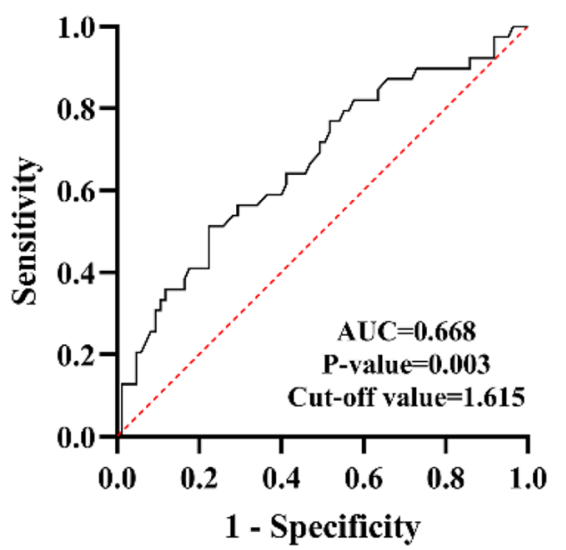

SIL-2R

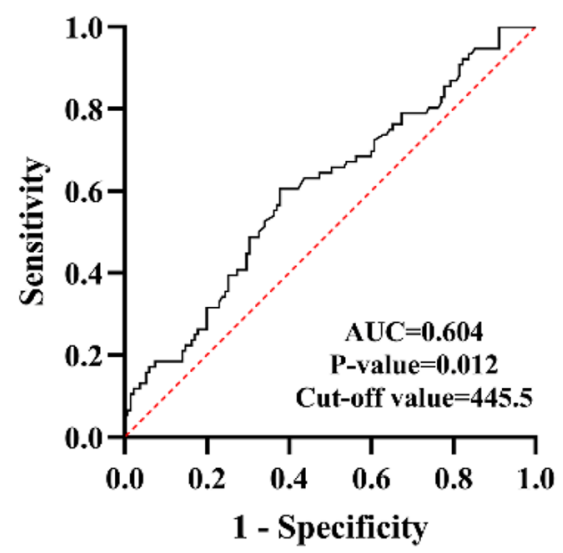

IL-8

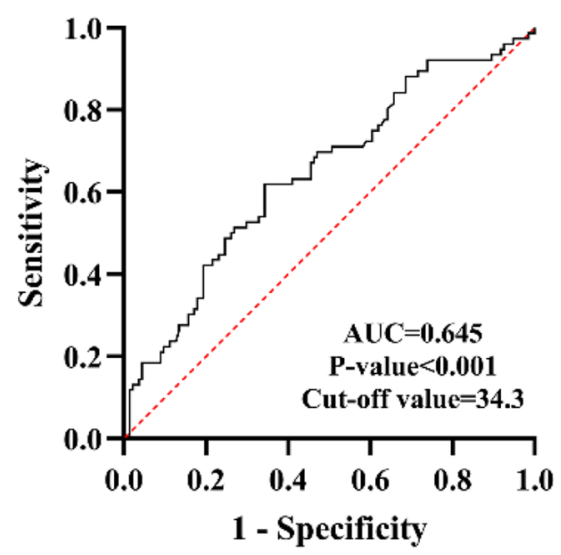

TNF- $\alpha$

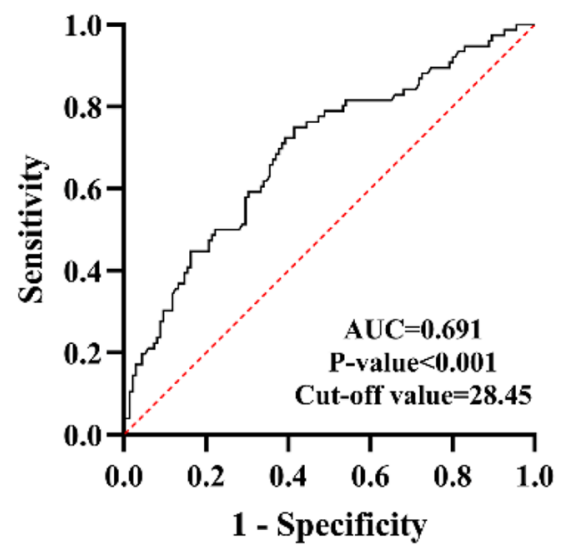

with previous researches, our study demonstrated that the inflammatory cytokines were significantly correlated with CSF occurrence after revascularization. However, the correlation coefficients of those cytokines with cTFC are smaller than other studies. It might result from the heterogeneity of population involved. Furthermore, compared with postPCI CSF, primary CSF phenomenon in previous studies was resulted from local inflammatory response without thrombus disorders, which accounts for the relatively weak relevance in our study. 
Fig. 3 Multivariate Logistic regression models of predictive factors for CSF. Multivariate Logistic regression models of predictive factors for CSF incorporating continuous variables (a) and dichotomized variables (b). The models were adjusted for gender, age, fast blood glucose, dilated blood pressure, heart rate, cardiac troponin $\mathrm{T}$, total cholesterol, high density lipoprotein, stent length and post dilation pressure. The risk of post-PCI CSF increases 1.120 fold per $1.0 \mathrm{mg} / \mathrm{l}$ increment of hs-CRP level and the risk increases 1.227 fold per $10 \mathrm{pg} / \mathrm{ml}$ increment of TNF- $\alpha$ (a). Continuous variables were dichotomized by cut-off value. The risk of post-PCI CSF increases 3.038 fold in patients with higher level of hs-CRP, the risk increases 2.103 fold in patients with higher level of sIL-2R and the risk increases 3.708 fold in patients with higher level of TNF- $\alpha(\mathbf{b})$

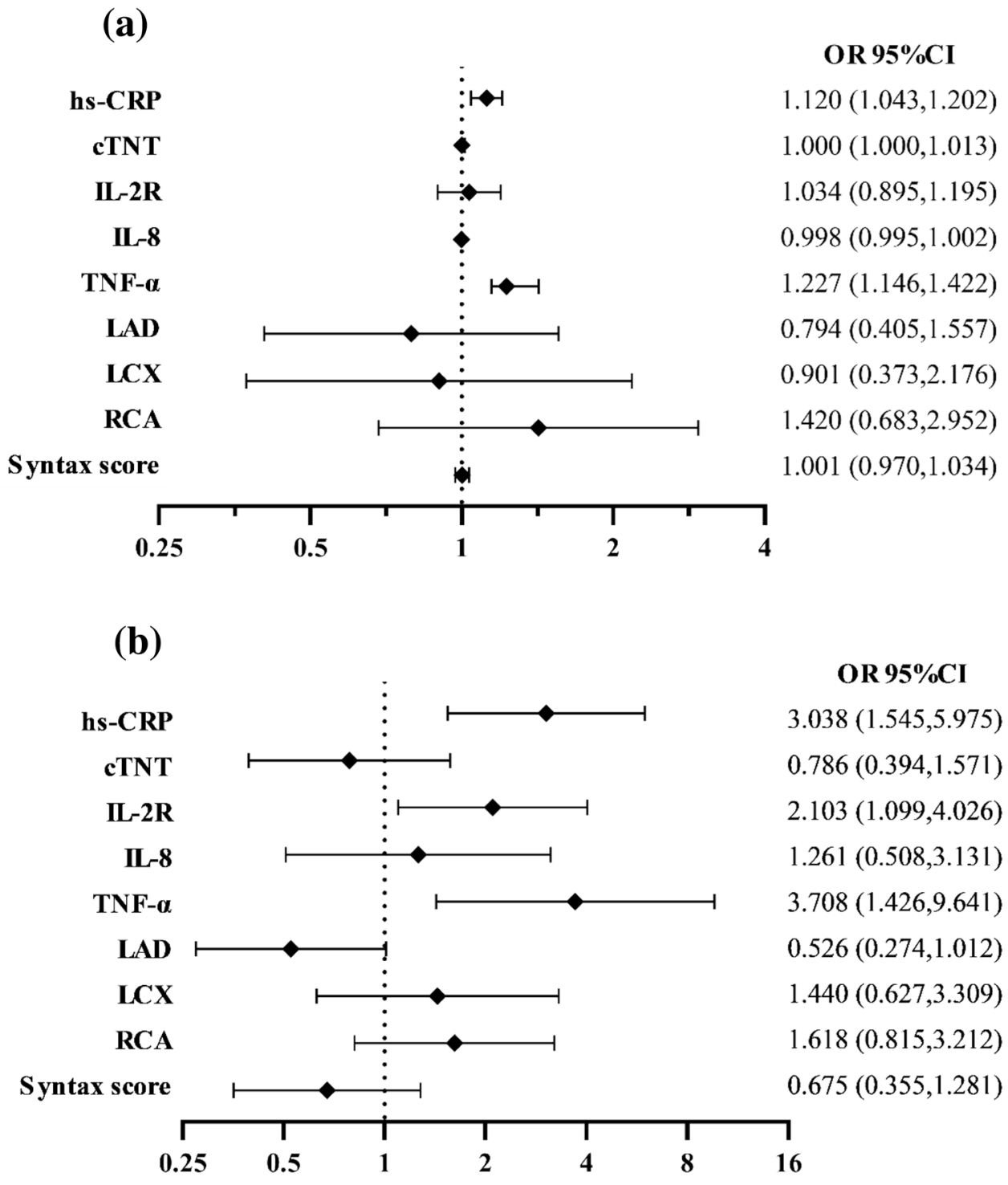

Accumulated evidences had reported that sustained high level of inflammatory cytokines was strongly linked to heavier thrombus burden and a greater extent of plaque instability [33]. An animal study demonstrated that CSF was resulted from impairment of microvascular integrity induced by altered expression level of IL-6 [34]. Previous experiment showed that another inflammatory cytokine, CRP, was released from vulnerable plaque and could intensify the local inflammatory response [11], which has the potential to predict CSF occurrence. And a large scale of clinical research provided evidence that hs-CRP, a more commonly measured index, is able to predict MACEs in patients with cardiovascular disease [35]. In addition, inflammatory cytokines released through paracrine from epicardial fat tissue would also aggravate CSF via affecting the endothelia function [36]. Furthermore, CSF could be improved by alleviating inflammatory responses regulated by mir-155
[37]. Furthermore. Similarly, in the present study, we have validated that inflammatory response plays an essential role in CSF phenomenon from clinical perspective. While other factors, including elder, hyperglycemia and higher stent to vessel ratio, showed non-significant impact. Collectively, inflammatory cytokines and CSF are inextricable. Patients with NSTE-ACS who had higher baseline level of inflammatory cytokines should be identified as individuals at high risk of developing post-PCI CSF.

Correction and treatment of causative factors are prerequisite for reducing occurrence of CSF during procedure. In PL-ACS registry trial, the mortality of ACS patients with final TIMI 0-2 after PCI was as high as $23.84 \%$ through 36-month follow-up [25]. Although traditional medication for improving the CSF/no-reflow followed revascularization were given, studies show that the occurrence of MACE in period of follow-up remained high, as $30.88 \%$ 
from an randomized controlled trial RECOVER [26] and $26.53-39.84 \%$ in other clinical researches [38, 39]. Promisingly, emerging researches on anti-inflammatory treatment targeting various cytokines, including anakinra [40], colchicine [41] and canakinumab [42, 43], in patients with coronary atherosclerosis disease (CAD) had made gratifying achievements on improving the prognosis [44]. Anti-inflammatory therapy has been becoming an effective and epochmaking approach that could greatly improve the prognosis of patients with CAD. Based on our results, we speculate that anti-inflammatory therapy locally or systemically during revascularization should reduce the occurrence of CSF in patients with NSTE-ACS. However, additional largescale clinical studies are needed to further assess the utility and safety of the treatment and long-term outcomes.

Besides, the predictor of CSF or no-reflow phenomenon has been under debate. In 2019, Mustafa et al. have published a study that investigated the association of no-reflow phenomenon and serum inflammatory biomarkers in patients with STEMI. They found serum CD40 ligand level, as an indicator platelet activation, could be a predictor of noreflow phenomenon [45]. While inflammatory biomarkers including hs-CRP and WBC count could not predict noreflow, which is inconsistent with our results. We believe such disparity could be attributed to the limited sample size of both studies, the difference in studied population and the difference between CSF and no-reflow. Based on current evidences, both inflammation and platelet activation play a role in CSF, and inflammation could interlay with platelet in a complicated mechanism that are yet not well understood. Further well-designed studies with larger sample size are needed to provide stronger evidence, and further researches on the relationship between inflammation and platelet activation are needed to unravel their underlying mechanism.

\section{Limitations}

The study is lack of concise morphological description of plaque which was usually measured using intravascular ultrasound. The mechanism of post-PCI CSF was more complicated than primary CSF due to various contributors. Further study is warranted to comprehensively explain the determinants of post-PCI CSF. Besides, we only validate common inflammatory cytokines, further studies thoroughly detecting inflammatory cytokines are necessitated. In addition, microcirculatory dysfunction has been proposed as a principal mechanism of CSF, which haven't been assessed in our study. Further researches on exploring the relationship between microcirculatory dysfunction and CSF are warranted.

\section{Conclusion}

Elevated circulating levels of inflammatory cytokine including hs-CRP, sIL-2R and TNF- $\alpha$ rather than differences of PCI related parameters could play a crucial role in predicting post-PCI CSF phenomenon in patients with NSTE-ACS. Our results indicate that anti-inflammatory therapy during revascularization could possibly be an effective prevention for CSF in patients with NSTE-ACS.

Supplementary Information The online version contains supplementary material available at https://doi.org/10.1007/s10554-022-02529-8.

Author contributions Conception and study design: YS, MW. Administrative support: YS, BM, MW, WC. Provision of study materials or patients: YS, YW, CW, YL, YY, KH, YL, WC, MW. Collection and assembly of data: YS, YW, CW. Data analysis and interpretation: YS, YW, CW, WC. Manuscript writing: YS, WC, MW. Final approval of manuscript: YS, YW, CW, YL, YY, KH, BM, YL, WC, MW.

Funding This research received no specific grant from any funding agency in the public, commercial.

Data availability The datasets used and/or analyzed during the current study are available from the corresponding author on reasonable request.

\section{Declarations}

Conflict of interest All authors state to have no associations with commercial entities that provided any support and could be viewed as having an interest in the general area of the submitted manuscript. All authors state to have no similar financial associations involving their spouse or their children under 18 years of age. All authors state that the submitted manuscript is not relevant to any non-financial association.

Ethical approval The study was approved by the Ethics Committee of Shanghai Tenth People's Hospital (SHSY-IEC-4.1/20-139/01), the Ethics Committee of Chongming Branch of Shanghai Tenth People's Hospital (SYCM-YJKT-20-0814/01) and the Ethics Committee of Putuo District Liqun Hospital (RT-202013).

Consent to participate Written informed consent for blood sample detection and medical treatment was acquired from each patient on admission.

Consent for publication Consent for publication was obtained from all authors.

Open Access This article is licensed under a Creative Commons Attribution 4.0 International License, which permits use, sharing, adaptation, distribution and reproduction in any medium or format, as long as you give appropriate credit to the original author(s) and the source, provide a link to the Creative Commons licence, and indicate if changes were made. The images or other third party material in this article are included in the article's Creative Commons licence, unless indicated otherwise in a credit line to the material. If material is not included in the article's Creative Commons licence and your intended use is not permitted by statutory regulation or exceeds the permitted use, you will need to obtain permission directly from the copyright holder. To view a copy of this licence, visit http://creativecommons.org/licenses/by/4.0/. 


\section{References}

1. Su Q, Li L, Liu Y (2013) Short-term effect of verapamil on coronary no-reflow associated with percutaneous coronary intervention in patients with acute coronary syndrome: a systematic review and meta-analysis of randomized controlled trials. Clin Cardiol 36:E11-E16

2. Ishihara M, Kojima S, Sakamoto T et al (2005) Acute hyperglycemia is associated with adverse outcome after acute myocardial infarction in the coronary intervention era. Am Heart $\mathbf{J}$ 150:814-820

3. Dong-bao L, Qi H, Zhi L, Shan W, Wei-ying J (2010) Predictors and long-term prognosis of angiographic slow/no-reflow phenomenon during emergency percutaneous coronary intervention for ST-elevated acute myocardial infarction. Clin Cardiol 33:E7-12

4. Dong L, Mintz GS, Witzenbichler B et al (2015) Comparison of plaque characteristics in narrowings with ST-elevation myocardial infarction (STEMI), non-STEMI/unstable angina pectoris and stable coronary artery disease (from the ADAPT-DES IVUS Substudy). Am J Cardiol 115:860-866

5. Mukhopadhyay S, Kumar M, Yusuf J, Gupta VK, Tyagi S (2018) Risk factors and angiographic profile of coronary slow flow (CSF) phenomenon in North Indian population: an observational study. Indian Heart J 70:405-409

6. Sanati H, Zahedmehr A, Firouzi A et al (2013) Coronary flow assessment in unstable angina/non ST-segment elevation myocardial infarction patients via thrombolysis in myocardial infarction frame count in angiography. Res Cardiovasc Med 2:95-98

7. Babat N, Kaya Y, Demir H (2019) Correlation IMA with TIMI frame count in slow coronary flow: can it be a guide for treatment? Aging Male 23:635

8. Riza Erbay A, Turhan H, Yasar AS et al (2005) Elevated level of plasma homocysteine in patients with slow coronary flow. Int $\mathrm{J}$ Cardiol 102:419-423

9. Turhan H, Saydam GS, Erbay AR et al (2006) Increased plasma soluble adhesion molecules; ICAM-1, VCAM-1, and E-selectin levels in patients with slow coronary flow. Int J Cardiol 108:224-230

10. Zhu YT, Zhu LP, Wang ZY et al (2020) Plasma choline as a diagnostic biomarker in slow coronary flow. Cardiol Res Pract 2020:7361434

11. Muller KA, Chatterjee M, Rath D, Geisler T (2015) Platelets, inflammation and anti-inflammatory effects of antiplatelet drugs in ACS and CAD. Thromb Haemost 114:498-518

12. Vaidya K, Martinez G, Patel S (2019) The role of colchicine in acute coronary syndromes. Clin Ther 41:11-20

13. Libby $P$ (2017) Interleukin-1 beta as a target for atherosclerosis therapy: biological basis of cantos and beyond. J Am Coll Cardiol 70:2278-2289

14. De Gennaro L, Brunetti ND, Montrone D, De Rosa F, Cuculo A, Di Biase M (2012) Subacute inflammatory activation in subjects with acute coronary syndrome and left ventricular dysfunction. Inflammation 35:363-370

15. Ikeda U, Ito T, Shimada K (2001) Interleukin-6 and acute coronary syndrome. Clin Cardiol 24:701-704

16. Didion SP, Kinzenbaw DA, Schrader LI, Chu Y, Faraci FM (2009) Endogenous interleukin-10 inhibits angiotensin II-induced vascular dysfunction. Hypertension 54:619-624

17. Beguin EP, van den Eshof BL, Hoogendijk AJ et al (2019) Integrated proteomic analysis of tumor necrosis factor alpha and interleukin 1beta-induced endothelial inflammation. J Proteomics 192:89-101

18. Roffi M, Patrono C, Collet JP et al (2016) 2015 ESC Guidelines for the management of acute coronary syndromes in patients presenting without persistent ST-segment elevation: Task Force for the Management of Acute Coronary Syndromes in Patients Presenting without Persistent ST-Segment Elevation of the European Society of Cardiology (ESC). Eur Heart J 37:267-315

19. Authors/Task Force m, Windecker S, Kolh P, et al (2014) ESC/ EACTS Guidelines on myocardial revascularization: the Task Force on Myocardial Revascularization of the European Society of Cardiology (ESC) and the European Association for CardioThoracic Surgery (EACTS) developed with the special contribution of the European Association of Percutaneous Cardiovascular Interventions (EAPCI). Eur Heart J 2014(35):2541-2619

20. Myler RK, Shaw RE, Stertzer SH et al (1992) Lesion morphology and coronary angioplasty: current experience and analysis. J Am Coll Cardiol 19:1641-1652

21. Ziada KM, Tuzcu EM, De Franco AC et al (1997) Intravascular ultrasound assessment of the prevalence and causes of angiographic "haziness" following high-pressure coronary stenting. Am J Cardiol 80:116-121

22. Schukro C, Gruska M, Syeda B et al (2007) Duration of development of symptomatic in-stent restenosis correlates with the stentto-vessel-diameter ratio: an intravascular ultrasound study. Coron Artery Dis 18:507-512

23. Gibson CM, Cannon CP, Daley WL et al (1996) TIMI frame count: a quantitative method of assessing coronary artery flow. Circulation 93:879-888

24. Kang SJ, Nakano M, Virmani R et al (2012) OCT findings in patients with recanalization of organized thrombi in coronary arteries. JACC Cardiovasc Imaging 5:725-732

25. Karwowski J, Polonski L, Gierlotka M et al (2016) Post-procedural TIMI flow grade 2 is not associated with improved prognosis in patients with non-ST-segment elevation myocardial infarction undergoing percutaneous coronary revascularization (PL-ACS registry). Cardiol J 23:402-410

26. Huang D, Qian J, Ge L et al (2012) REstoration of COronary flow in patients with no-reflow after primary coronary interVEntion of acute myocaRdial infarction (RECOVER). Am Heart $\mathbf{J}$ 164:394-401

27. Yurtdas M, Yaylali YT, Kaya Y, Ozdemir M (2014) Increased plasma high-sensitivity C-reactive protein and myeloperoxidase levels may predict ischemia during myocardial perfusion imaging in slow coronary flow. Arch Med Res 45:63-69

28. Li JJ, Qin XW, Li ZC et al (2007) Increased plasma C-reactive protein and interleukin-6 concentrations in patients with slow coronary flow. Clin Chim Acta 385:43-47

29. Faramarz-Gaznagh S, Rasmi Y, Khadem-Ansari MH et al (2016) Transcriptional activity of gene encoding subunits R1 and R2 of interferon gamma receptor in peripheral blood mononuclear cells in patients with slow coronary flow. J Med Biochem 35:144-149

30. Liang Q, Lei X, Huang X, Fan L, Yu H (2021) Elevated lipoprotein-associated phospholipase A2 is valuable in prediction of coronary slow flow in non-ST-segment elevation myocardial infarction patients. Curr Probl Cardiol 46:100-596

31. Ucgun T, Basar C, Memisogullari R, Demirin H, Turker Y, Aslantas Y (2014) Serum visfatin and omentin levels in slow coronary flow. Rev Port Cardiol 33:789-794

32. Sigirci S, Sarikaya R, Keskin K, et al (2019) Can biomarkers help us to understand the pathogenesis of coronary slow flow? Endocan and omentin-I in slow coronary flow phenomenon. Turk Kardiyol Dern Ars 47:251-257

33. Wu X, Zhang Y, Wu Z et al (2016) Plasma lipoprotein-associated phospholipase A2 level is an independent predictor of high thrombus burden in patients with acute ST-segment elevation myocardial infarction. Int Heart J 57:689-696

34. Bramos D, Ikonomidis I, Tsirikos N et al (2008) The association of coronary flow changes and inflammatory indices to ischaemiareperfusion microvascular damage and left ventricular remodelling. Basic Res Cardiol 103:345-355 
35. Emerging Risk Factors C, Kaptoge S, Di Angelantonio E et al (2010) C-reactive protein concentration and risk of coronary heart disease, stroke, and mortality: an individual participant meta-analysis. Lancet 375:132-140

36. Weferling M, Vietheer J, Keller T, Fischer-Rasokat U, Hamm CW, Liebetrau C (2021) Association between primary coronary slowflow phenomenon and epicardial fat tissue. J Invasive Cardiol 33:E59-E64

37. Huang J, Yang Q, He L, Huang J (2018) Role of TLR4 and miR155 in peripheral blood mononuclear cell-mediated inflammatory reaction in coronary slow flow and coronary arteriosclerosis patients. J Clin Lab Anal 32:e22232

38. Zhang HCY, Wu Z (2010) Effect of intracoronary verapamil on no-reflow during percutaneous coronary intervention. Shangdong Med J 50:23

39. Wu MYZ (2012) Clinical efficacy and safety of intracoronary verapamil during percutaneous coronary intervention in patients with acute coronary syndromes. Chin J Geriatr 31:4

40. Abbate A, Kontos MC, Abouzaki NA et al (2015) Comparative safety of interleukin-1 blockade with anakinra in patients with ST-segment elevation acute myocardial infarction (from the VCUART and VCU-ART2 pilot studies). Am J Cardiol 115:288-292

41. Martinez GJ, Robertson S, Barraclough J et al (2015) Colchicine acutely suppresses local cardiac production of inflammatory cytokines in patients with an acute coronary syndrome. J Am Heart Assoc 4:e002128

42. Ridker PM, Libby P, MacFadyen JG et al (2018) Modulation of the interleukin- 6 signalling pathway and incidence rates of atherosclerotic events and all-cause mortality: analyses from the Canakinumab Anti-Inflammatory Thrombosis Outcomes Study (CANTOS). Eur Heart J 39:3499-3507

43. Ridker PM, Everett BM, Thuren T et al (2017) Antiinflammatory therapy with canakinumab for atherosclerotic disease. N Engl J Med 377:1119-1131

44. Wudexi I, Shokri E, Abo-Aly M, Shindo K, Abdel-Latif A (2021) Comparative effectiveness of anti-inflammatory drug treatments in coronary heart disease patients: a systematic review and network meta-analysis. Mediators Inflamm 2021:5160728

45. Tascanov MB, Tanriverdi Z, Gungoren F et al (2019) Association between the no-reflow phenomenon and soluble CD40 ligand level in patients with acute ST-segment elevation myocardial infarction. Medicina (Kaunas) 55:376

Publisher's Note Springer Nature remains neutral with regard to jurisdictional claims in published maps and institutional affiliations. 